\title{
High-Risk Repair of Traumatic Mitral Valve Rupture in the Setting of Polytrauma
}

\author{
Michael John Paisley ${ }^{1}$ Zachary Deboard ${ }^{2}$ Donald Thomas ${ }^{3}$ \\ ${ }^{1}$ Department of Surgery, Santa Barbara Cottage Hospital, Santa \\ Barbara, California, United States \\ 2 Department of Cardiothoracic Surgery, University of Utah Hospital, \\ Salt Lake City, Utah, United States \\ ${ }^{3}$ Division of Cardiothoracic Surgery, Department of Surgery, Oregon

\begin{abstract}
Address for correspondence Michael John Paisley, Department of Surgery, Santa Barbara Cottage Hospital, 400 W Pueblo Street, Santa Barbara, CA 93102-0689, United States
\end{abstract} \\ (e-mail: mjpaisley@gmail.com).
} Health and Science University, Portland, Oregon, United States

Thorac Cardiovasc Surg Rep 2018;7:e1-e3.

\begin{abstract}
Keywords

- MV surgery

- trauma

- shock: systemic

- cardiac or circulatory

- myocardial injury: blunt

- penetrating

- and iatrogenic

Background The rare complication of mitral valve rupture from blunt trauma is certainly not at the top of the differential of shock.

Case Description We report the case of a 56-year-old woman who sustained numerous injuries after a 30-m fall with cardiogenic shock secondary to mitral valve rupture causing severe mitral valve regurgitation. Management included successful primary leaflet repair, annuloplasty, and single vessel coronary artery bypass.

Conclusion Valvular repair in the setting of polytrauma is a complex decision that requires careful balance of risks and benefits relative to patient stability.
\end{abstract}

\section{Introduction}

Cardiac valvular injury after blunt trauma is an unusual etiology of shock and merits a high index of suspicion for diagnosis. The majority of mitral valve (MV) injuries involve the papillary muscles or chordae tendinae with repair tailored at the underlying pathology. Few reports of MV injury in the setting of trauma exist with the largest review totaling 82 cases in a 2009 publication. ${ }^{1}$ We report a case of cardiogenic shock due to MV insufficiency in setting of polytrauma.

\section{Case Description}

A 56-year-old woman with a history of depression presented after a fall of $30 \mathrm{~m}$, reportedly landing upon bushes cushioning her impact. Admission blood pressure was $88 / 48 \mathrm{~mm} \mathrm{Hg}$ and pulse was $110 \mathrm{bpm}$. Physical examination and chest radiograph were unremarkable for any significant signs of trauma. Cross-sectional imaging revealed multiple rib fractures, right pneumothorax, splenic, renal and liver lacera- tions, cervical and thoracic spine fractures, an ulnar fracture, and a spinal epidural hematoma (SEH). Her calculated injury severity score was 29 and trauma score-injury severity score predicting $24.4 \%$ likelihood of death. ${ }^{2}$

A 28 French right-sided chest tube yielded less than $200 \mathrm{cc}$ of blood and her hemodynamics continued to indicate shock. Transfusion of four units red blood cells failed to stabilize the patient and a norepinephrine infusion yielded transient improvement. Remarkable laboratory results included troponin-I of $10.5 \mathrm{mg} / \mathrm{mL}$ and lactate of $3.4 \mathrm{mmol} / \mathrm{L}$. Her hemodynamics deteriorated and a pulmonary artery (PA) catheter demonstrated PA pressures of $44 / 26 \mathrm{~mm} \mathrm{Hg}$, and cardiac index $(\mathrm{CI})$ of $0.9 \mathrm{~L} / \mathrm{min} / \mathrm{m}^{2}$. Dobutamine was initiated.

Transthoracic echocardiography demonstrated severe mitral regurgitation with intact left ventricular function. An intra-aortic balloon pump (IABP) was placed resulting in improvement of $\mathrm{CI}$ to $2.2 \mathrm{~L} / \mathrm{min} / \mathrm{m}^{2}$. Transesophageal echocardiogram (TEE) revealed a defect in the posterior MV involving P1, P2, and the annulus suspicious for the involvement of the circumflex artery (-Fig. 1). Coronary angiography received

November 9, 2017

accepted

December 18, 2017
DOI https://doi.org/

10.1055/s-0037-1621747. ISSN 2194-7635. (c) 2018 Georg Thieme Verlag KG
Stuttgart · New York

License terms

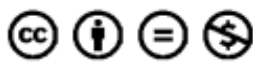




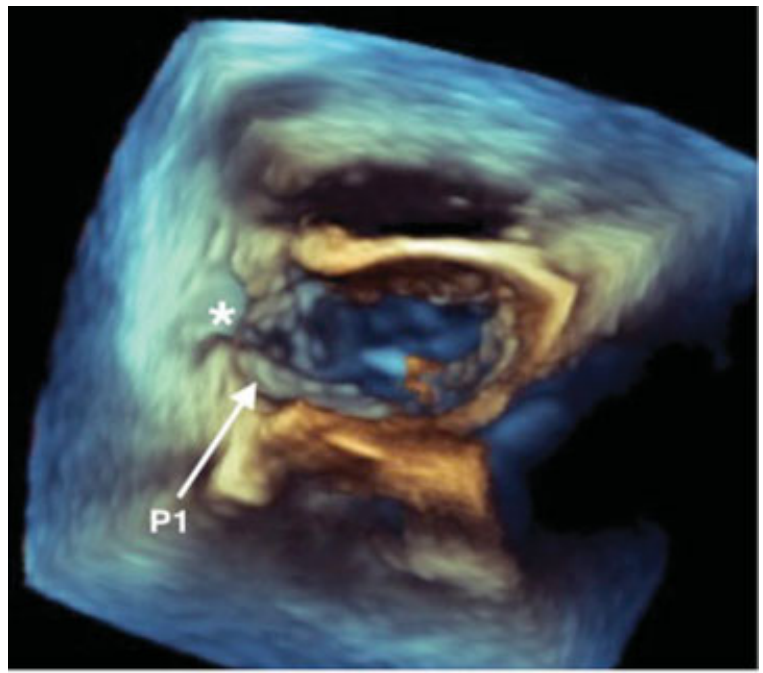

Fig. 1 Preoperative three-dimensional transesophageal echocardiogram demonstrating posterior leaflet injury $\left({ }^{*}\right)$.

was performed demonstrating irregularity and disruption with systolic compression of the proximal circumflex artery. Despite high risk, she was taken to the operating room 41 hours after presentation.

Via standard sternotomy, exposure of the heart showed dilation of both atria and ventricles and an area of ecchymosis about the proximal circumflex artery. After institution of cardiopulmonary bypass (CPB) via aortic and bicaval cannulation, a trans-septal approach exposed the MV. A flail segment of P1 was noted with a tear extending across P1 into the annulus and left posterior atrial wall. The valve leaflet was reapproximated with interrupted 4-0 Prolene. A pledgeted repair was used for the annulus and posterior left atrial wall. A $30 \mathrm{~mm}$ Profile 3D annuloplasty ring (Medtronic, Minneapolis, Minnesota, United States) was placed. Excellent valvular competence was achieved via pressure testing. Finally, a coronary artery bypass (CAB) to the obtuse marginal one was performed with a reverse saphenous vein graft. The patient was liberated from CPB, with TEE confirming excellent valve function, and the operation completed.

Dobutamine and the IABP were discontinued 3 hours postoperatively. Respiratory failure required intubation for 9 days with diuresis and antibiotics for pansensitive pseudomonas pneumonia. Her cardiac recovery was otherwise uneventful. Management of her orthopedic injuries was uncomplicated. She was discharged to a rehabilitation facility on hospital day 23 and is doing well 12 months later.

\section{Discussion}

Cardiac valve injury after blunt chest trauma is rare with the aortic valve injured most commonly followed by mitral and tricuspid valves. ${ }^{1,3}$ The mechanism of MV injury in blunt trauma is postulated to be high-velocity deceleration causing increased intracardiac pressure during end diastole and early systole coinciding with the moment of trauma. ${ }^{1}$ Surgical options for traumatic MV incompetence include repair and replacement and depend on the nature of anatomic defect, patient stability, surgeon preference. Papillary muscle rupture is the most common etiology of traumatic MV insufficiency and is more often approached with valve replacement, followed by chordae tendinae lesions, which are more frequently but not exclusively approached by repair. Leaflet injuries are the least common location with method of intervention favoring neither repair nor replacement. Overall, operative approach is more commonly replacement over repair and is likely due to the higher incidence of papillary muscle injury making repair more difficult, but many variables are considered in deciding the most appropriate intervention. ${ }^{1}$

Our relatively young patient's intact and viable papillary and perivalvular tissue and intact chordae influenced us to perform repair with annuloplasty and CA to correct circumflex artery dissection. To our knowledge and based on literature review, this is the first reported case of primary leaflet repair with ring annuloplasty and $\mathrm{CAB}{ }^{4}$

Echocardiogram remains the gold-standard diagnostic tool in mitral insufficiency, aiding in anatomic evaluation and guiding operative management. ${ }^{4,5}$ Despite being a common tool for hemodynamic support in mitral insufficiency, there are no reports regarding the use of IABP in trauma. Our patient's shock was temporized with IABP, allowing time for a multidisciplinary approach to evaluate her other injuries including her coronary artery injury leading to the decision to offer cardiac surgery.

Solid organ lacerations and SEH pose a potential risk for worsening hemorrhage during systemic heparinization. However, Santaniello et al have demonstrated that patients undergoing CPB with grade 1 and 2 splenic and liver injuries are not subject to a greater risk of hemorrhagic conversion. ${ }^{6}$ There is a paucity of literature for the risk of clinically significant progression of a previously identified traumatic epidural hematoma after systemic heparinization, though there are reports of clinically significant spontaneous SEH after CPB for MV surgery. ${ }^{7}$ Given risk of spontaneous hematoma development during $\mathrm{CPB}$, progression of an existing SEH should be considered in the decision to anticoagulate.

In patients with acute severe mitral insufficiency from traumatic causes, the timing of surgery is based on the patient's hemodynamic stability. Should inotropes and afterload reduction lead to hemodynamic stability and adequate perfusion, operative repair may be delayed. However, in the unstable patient the repair may have to be performed expeditiously weighing the risks and benefits carefully. ${ }^{1}$

\section{Conflict of Interest}

None.

\section{References}

1 Pasquier M, Sierro C, Yersin B, Delay D, Carron PN. Traumatic mitral valve injury after blunt chest trauma: a case report and review of the literature. J Trauma 2010;68(01):243-246

2 Baker SP, O'Neill B, Haddon W Jr, Long WB. The injury severity score: a method for describing patients with multiple injuries and evaluating emergency care. J Trauma 1974;14(03):187-196

3 Turan AA, Karayel FA, Akyildiz E, et al. Cardiac injuries caused by blunt trauma: an autopsy based assessment of the injury pattern. J Forensic Sci 2010;55(01):82-84 
4 Kumagai H, Hamanaka Y, Hirai S, Mitsui N, Kobayashi T. Mitral valve plasty for mitral regurgitation after blunt chest trauma. Ann Thorac Cardiovasc Surg 2001;7(03):175-179

5 Shaikh N, Ummunissa F, Adbel Sattar M. Traumatic Mitral Valve and Pericardial Injury. Case Reports in Critical Care. (Sept 2013). Online: www.hindawi.com/journals/cricc/. Accessed December 31, 2017
6 Santaniello JM, Miller PR, Croce MA, et al. Blunt aortic injury with concomitant intra-abdominal solid organ injury: treatment priorities revisited. J Trauma 2002;53(03):442-445, discussion 445

7 Karlekar A, Dutta D, Dev Arora K, Mishra YK. Spinal-epidural hematoma presenting as paraplegia following mitral valve surgery: a case report. J Cardiothorac Vasc Anesth 2015;29(01):139-141 